\title{
NUMERICAL DYNAMICS OF ORDINARY DIFFERENTIAL EQUATIONS WITH SINGULARITY
}

\author{
KEVIN HOCKETT
}

(Communicated by Charles Pugh)

\begin{abstract}
We investigate the global dynamics of Euler's method and second order Runge-Kutta when applied to certain nonlinear ordinary differential equations that possess a singularity. We show that the numerics admit spurious 'chaotic' dynamics in the form of subshifts of finite type with positive topological entropy independent of the choice of stepsize. We show that using a higher order method can, in fact, increase the topological entropy of the numerical dynamics. Techniques from complex analytic dynamics give some insight into this phenomenon.
\end{abstract}

\section{INTRODUCTION}

There has recently been a great deal of interest in understanding the global dynamics of the classical algorithms of analysis. Among such investigations are the dynamics of Newton's method (Curry, Garnett, and Sullivan [CGS], Smale [Sm]), the classical QR algorithm (Shub and Vasquez [SV]), Rayleigh quotient iteration (Batterson and Smillie [BS]), and 'computational chaos' occurring when the stepsize of a numerical algorithm is taken too large (Lorenz [Lo], Sweby, Ye, and Griffiths [SwYG]).

In Hockett [Ho] we investigated the global numerical dynamics of Euler's method $E_{h}$ when applied to the central force problem. There it is shown that for all $h>0$ the nonwandering set of $E_{h}$ contains a subset on which the dynamics of $E_{h}$ are topologically conjugate to a subshift of finite type having positive topological entropy.

This paper examines the global dynamics of several numerical schemes when applied to ordinary differential equations of the form

$$
\dot{x}=f(x) \text {. }
$$

Here $x \in \mathbf{R}^{n}$ and we begin by examining Euler's method $E_{h}$ with stepsize $h$, considering first the dynamics of $E_{h}$ when applied to a gradient dynamical system on $\mathbf{R}^{n}$. As indicated above, it is well known that numerical algorithms can yield spurious chaotic behavior if the stepsize is not chosen appropriately [Lo, SwYG]. In the case of gradient systems, however, the situation is quite

Received by the editors June 3, 1991.

1991 Mathematics Subject Classification. Primary 34C35, 58F03, 39B12.

Research supported in part by the GWU University Facilitating Fund. 
good in terms of numerical stability. We show that if the potential function for the gradient system is sufficiently smooth, then it is always possible to choose a stepsize $h$ so that the numerical approximation preserves certain qualitative features of the original gradient system. In particular, one can always eliminate spurious periodic points or spurious 'chaos'.

On the other hand, if the potential function of a gradient system has a singularity, it is possible that no choice of stepsize will produce a numerical approximation with qualitative properties resembling those of the original system. Indeed, we construct an example that yields a result similar to that in [Ho]: for all $h>0$ the nonwandering set of $E_{h}$ contains a set on which the dynamics of $E$ are topologically semiconjugate to a subshift of finite type with positive topological entropy. This result offers a basis for comparison to the numerical dynamics of other algorithms, and we next investigate the global dynamics of second order Runge-Kutta $R_{h}$. We show that rather than yielding a better approximation to the dynamics of the underlying ODE, the nonwandering set of $R_{h}$ admits a subshift of finite type with larger topological entropy than the entropy of $E_{h}$. Thus, loosely speaking, the numerical dynamics for $R_{h}$ are 'more chaotic' than for $E_{h}$.

Some insight into this phenomenon can be gained by complexifying equation (1.1), and in the next section we study systems on the plane of the form

$$
\dot{z}=f(z),
$$

where $f(z)$ is a meromorphic function of $z \in C$. In this case the iterated mapping obtained by applying a single step method to (1.2) is a rational map that can be analyzed using techniques from complex analytic dynamics.

\section{Symbolic DYNAmics}

Symbolic dynamics is a basic tool in the analysis that follows, so we recall here the basic definitions and results. See, e.g., Bowen [Bo] or Shub [Sh] for a more complete discussion. Let $f: X \rightarrow X$ be a continuous mapping of a topological space $X$. A point $x \in X$ is wandering if there exists a neighborhood $U$ of $x$ such that $f^{k}(U) \cap U=\varnothing$ for all $k \geq 1$. A point that does not wander is called nonwandering, and the nonwandering set $\Omega_{f}$ (the set of all nonwandering points) is closed and satifies $f\left(\Omega_{f}\right) \subset \Omega_{f}$.

Let $S_{n}=\{1,2, \ldots, n\}$ with the discrete topology, and let $\Sigma_{n}=\prod_{0}^{\infty} S_{n}$ with the product topology. $\Sigma_{n}$ is the space of semi-infinite sequences of the symbols $S_{n}$. The space $\Sigma_{n}$ is metrizable, and the metric $d(a, b)=\Sigma_{k=0}^{\infty}\left|a_{k}-b_{k}\right| / 2^{k+1}$ induces the product topology on $\Sigma_{n}$. We define the shift map $\sigma: \Sigma_{n} \rightarrow \Sigma_{n}$ by $\sigma\left(a_{0} a_{1} a_{2} \cdots\right)=a_{1} a_{2} \cdots$, which is easily seen to be a continuous $n$-to1 map of $\Sigma_{n}$. Now let $\mathscr{A}$ be a square matrix of 0 's and 1 's, and define $\Sigma_{\mathscr{A}}=\left\{a=a_{0} a_{1} a_{2} \cdots \in \Sigma_{n} \mid \mathscr{A}_{a_{i}, a_{i+1}}=1, i=1,2, \ldots\right\}$. Letting $\sigma_{\mathscr{A}}$ denote the restriction of $\sigma$ to $\Sigma_{\mathscr{A}}$, we call the pair $\left(\Sigma_{\mathscr{A}}, \sigma_{\mathscr{A}}\right)$ a subshift of finite type. The matrix $\mathscr{A}$ is called the transition matrix of the subshift. $\mathscr{A}$ can be shown to have at least one positive eigenvalue $\lambda$ (Gantmacher [Ga]).

Finally we recall the definition of topological entropy. We shall assume that $X$ is a compact metric space. A set $S \subset X$ is $(n, \varepsilon)$-separated for $f$ if $x, y \in S$ implies $d\left(f^{k}(x), f^{k}(y)\right)>\varepsilon$ for some $0 \leq k<n$. Let $s(n, \varepsilon)=\max \{\operatorname{card} S \mid S$ is $(n, \varepsilon)$-separated for $f\}$ and define the growth rate $h(f, \varepsilon)=\lim \sup _{n \rightarrow \infty} \ln s(n, \varepsilon) / n$. The topological entropy of $f$ is $h(f)=$ 
$\lim _{\varepsilon \rightarrow 0} h(f, \varepsilon)$. In general, $h(f)$ is difficult to compute. But for a subshift of finite type we have $h\left(\sigma_{\mathscr{A}}\right)=\ln \left(\lambda^{*}\right)$, where $\lambda^{*}$ is the largest eigenvalue of the transition matrix $\mathscr{A}[\mathrm{Bo}]$.

\section{GRADIENT SYSTEMS AND EULER'S METHOD}

Before we consider our first example we examine the numerical properties of gradient systems on $\mathbf{R}^{n}$, i.e., systems of the form

$$
\dot{x}=f(x)=-\operatorname{grad} V(x),
$$

where $x \in \mathbf{R}^{n}$ and $V: \mathbf{R}^{n} \rightarrow \mathbf{R}$. We will assume that $V$ is $C^{2}$ on all of $\mathbf{R}^{n}$. Note that any ODE of the form (1.1) with $f: R \rightarrow R$ a $C^{1}$ function may be regarded as such a gradient system with $V(x)=-\int f(x) d x$. If we apply Euler's method with stepsize $h$ to (3.1) we obtain the map

$$
x \stackrel{E_{h}}{\longrightarrow} x-h \operatorname{grad} V(x)
$$

which one iterates to obtain a numerical approximation to the solution of (3.1) based at $x$. Gradient systems without singularities have relatively simple dynamics (a 'typical' trajectory tends to an equilibrium point or tends to infinity) and we shall see that essentially the same is true of the numerical approximation generated by $E_{h}$.

We adopt the following notation. As above we shall write $f(x)=-\operatorname{grad} V(x)$, and let $A_{x}=D f(x)$, i.e., $A_{x}$ is the Hessian matrix of $-V(x)$. Finally, let $\bar{x}$ denote an equilibrium point of (3.1), i.e., a point for which $-\operatorname{grad} V(\bar{x})=0$. Hence $\bar{x}$ is a critical point of $V$.

Proposition 3.1. Let $\bar{x}$ be an equilibrium point of (3.1). Then $\bar{x}$ is a fixed point of $E_{h}$. Moreover, if $\lambda$ is an eigenvalue of $A_{\bar{x}}$ then $\mu=1+h \lambda$ is an eigenvalue of $D E_{h}(\bar{x})$.

Proof. The first statement follows since $E_{h}(\bar{x})=\bar{x}-h \operatorname{grad} V(\bar{x})=\bar{x}$. Next, let $v$ be an eigenvector of $A_{\bar{x}}$ corresponding to the eigenvalue $\lambda$. Then $D E_{h}(\bar{x}) v=$ $v+h A_{\bar{x}} v=v+h \lambda v=\mu v$.

Corollary 3.2. If $\bar{x}$ is a hyperbolic equilibrium point of (3.1) (all eigenvalues of $A_{\bar{x}}$ have nonzero real part) then for $h$ sufficiently small, $\bar{x}$ is a hyperbolic fixed point of $E_{h}$ (no eigenvalue of $D E_{h}(\bar{x})$ has unit modulus).

Thus if $\bar{x}$ is, for example, a hyperbolic sink for the system (3.1), then $\bar{x}$ is a hyperbolic sink for $E_{h}$ provided $h$ is chosen sufficiently small; however, we can say somewhat more. A well-known property of gradient systems is that the solution trajectory passing through a regular point of $V$ crosses the level curves of $V$ orthogonally (see, e.g., Hirsch and Smale [HS]). Part of this result is true for the numerical approximation that $E_{h}$ yields.

Proposition 3.3. For all regular points $x$ of $V$ and for $h$ sufficiently small (depending, in general, on $x), V\left(E_{h}(x)\right)<V(x)$.

Proof. Recall that $f(x)=-\operatorname{grad} V(x)$, and observe

$$
V\left(E_{h}(x)\right)-V(x)=V(x+h f(x))-V(x)=-h|\operatorname{grad} V(x)|^{2}+\mathscr{O}\left(h^{2}\right),
$$

the last equality being obtained from the Taylor expansion of $V(x+h f(x))$ about $h=0$. 


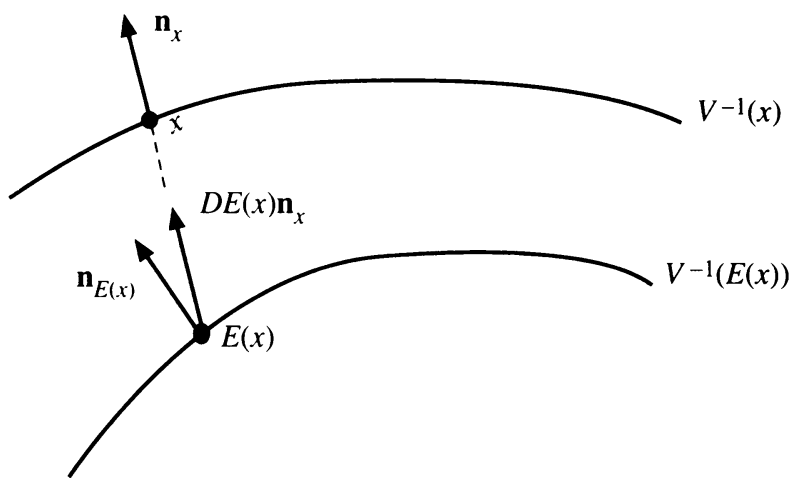

FigURE $0 . D E_{h}(x) \mathbf{n}_{x} \neq \alpha \mathbf{n}_{E_{h}(x)}$.

Thus in any compact region $K \subset \mathbf{R}^{n}$ for which the iterates $E_{h}^{n}(x)$ lie in $K$, we can choose a stepsize $h$ such that $V\left(E_{h}^{n}(x)\right)$ is decreasing along the $E_{h}$-orbit of $x$. As a result we have

Corollary 3.4. For $h>0$ sufficiently small, isolated minima of $V$ are asymptotically stable fixed points of $E_{h}$.

In particular, if we apply Euler's method to a single ODE of the form (1.1) with $f(x)$ a $C^{1}$ function on $\mathbf{R}$, then $h$ can always be chosen so that the fixed points of $E_{h}$ have the same stability properties as the equilibria of (1.1) and no spurious fixed points or periodic points exist for $E_{h}$. Thus, for example, if $f(x)$ is a polynomial (as, e.g., in [SwYG]) one can always eliminate any spurious periodic orbits by choosing $h$ sufficiently small.

It is not generally the case that the numerical trajectories 'cross the level sets of $V$ orthogonally'. One needs to make sense of the notion of a discrete trajectory crossing a level set orthogonally. Since $\mathbf{n}_{z}=\operatorname{grad} V(z)$ is normal to the level surface $V^{-1}(z)$, a reasonable requirement is that $D E_{h}(x) \mathbf{n}_{x}=\alpha \mathbf{n}_{E_{h}(x)}$ where $\alpha$ is a nonzero real number; that is, we require that the linearization of $E_{h}$ at $x$ carry the normal vector $\mathbf{n}_{x}$ determined by the gradient vector field at $x$ to a normal vector $\alpha \mathbf{n}_{E_{h}(x)}$ at $E_{h}(x)$. Since (for a gradient system) Euler's method proceeds from $x$ to $E_{h}(x)$ by following the normal direction $\mathbf{n}_{x}$ some distance, the above equation will not hold in general (see Figure 0).

\section{EULER vs. SECOND ORDER RUNGE-KutTA}

The above results do not hold in the case where $f(x)$ has singularities. Consider, for example, the ODE

$$
\dot{x}=1-\frac{1}{x}
$$

defined on $\mathbf{R}-\{0\}$. The phase portrait and integral curves for (4.1) are shown in Figure 1. Any solution with initial data $x(0)=x_{0} \in(1, \infty)$ satisfies $\lim _{t \rightarrow \infty} x(t)=\infty, \quad x=1$ is an unstable equilibrium point, and solutions with $x_{0} \in(-\infty, 1)-\{0\}$ hit the singular point at $x=0$ in finite time.

If we apply Euler's method with stepsize $h$ to (4.1), we obtain the map

$$
x \stackrel{E_{h}}{\longrightarrow} x+h(1-1 / x) .
$$




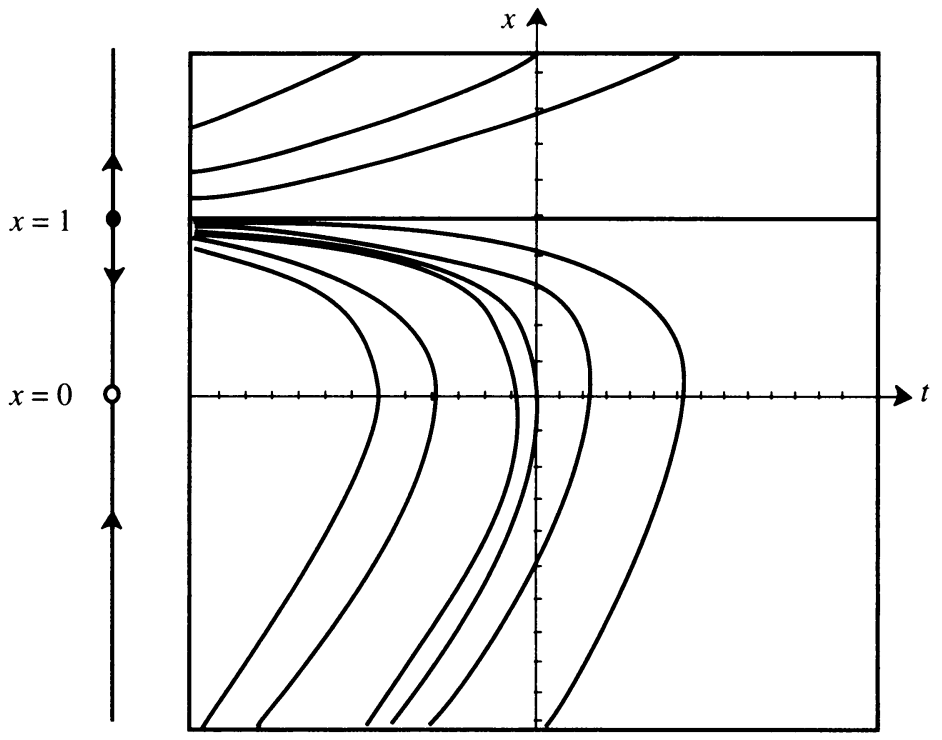

FIgURE 1. The phase portrait and integral curves of (4.1).

The numerical approximation of a solution of (4.1) is obtained by choosing $x_{0} \in \mathbf{R}-\{0\}$ and iterating $E_{h}$. Note that the map (4.2) has an unstable fixed point at $x=1$, so this local property of the flow is preserved by $E_{h}$; however, the dynamics of $E_{h}$ are much more complicated than the dynamics of the flow.

Theorem 4.1. For all $h>0$ the nonwandering set of the map $E_{h}$ of (4.2) contains a subset $\Lambda$ such that $\left.E_{h}\right|_{\Lambda}$ is topologically semiconjugate to the subshift of finite type with transition matrix

$$
\mathscr{A}=\left[\begin{array}{ll}
0 & 1 \\
1 & 1
\end{array}\right] ;
$$

that is, there exists a continuous surjective map $\varphi$ such that the following diagram commutes:

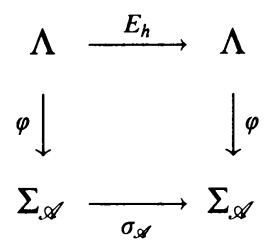

Moreover, $\left(\Sigma_{\mathscr{A}}, \sigma_{\mathscr{A}}\right)$ has topological entropy $h\left(\sigma_{\mathscr{A}}\right)=\ln \left(\frac{1+\sqrt{5}}{2}\right)$, hence $h\left(E_{h}\right) \geq$ $\ln \left(\frac{1+\sqrt{5}}{2}\right)$.

Proof. To prove the theorem we construct a pair of closed intervals $I_{1}, I_{2}$ satisfying

$$
E_{h}\left(I_{1}\right) \supset I_{2}, \quad E_{h}\left(I_{2}\right) \supset I_{1} \cup I_{2} .
$$

Let $\Lambda_{n}=\left(I_{1} \cup I_{2}\right) \cap E_{h}^{-n}\left(I_{1} \cup I_{2}\right)$. By (4.3) each $\Lambda_{n}$ is a nonempty compact subset of $I_{1} \cup I_{2}$, and these sets satisfy $\Lambda_{n} \supset \Lambda_{n+1}$. Thus the desired set $\Lambda=\bigcap_{n=0}^{\infty} \Lambda_{n}$ 


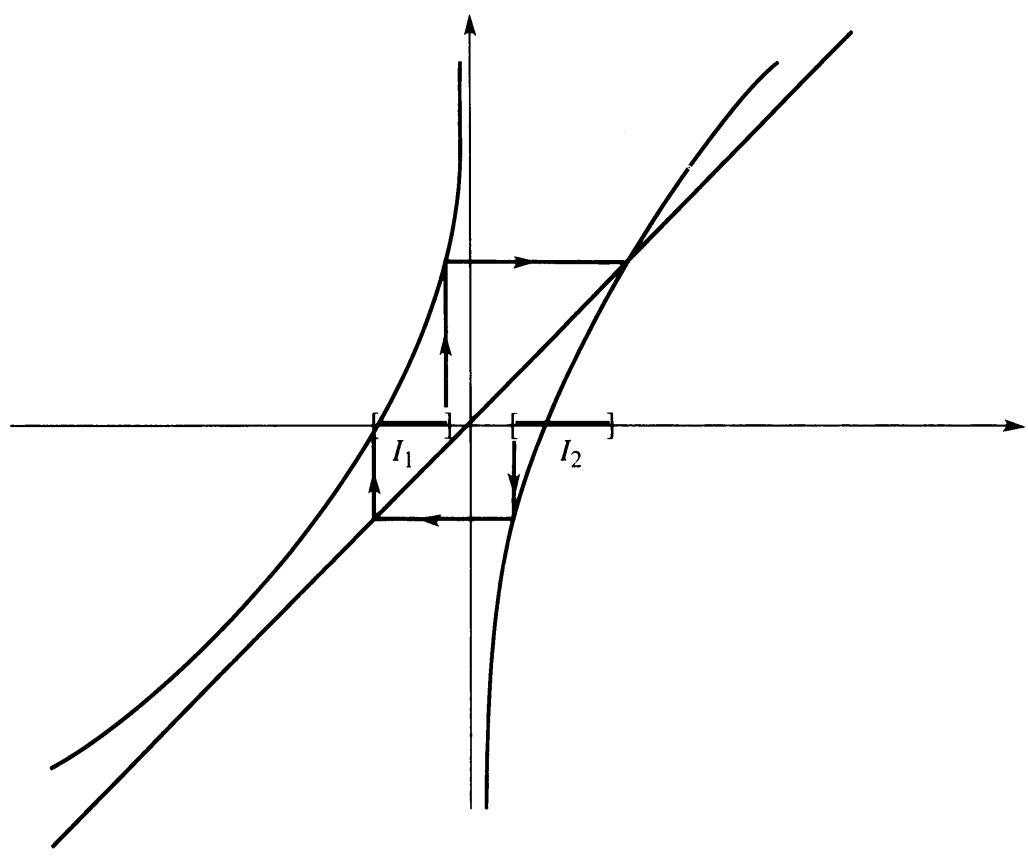

FIGURE 2. Construction of the intervals $I_{1}, I_{2}$.

is nonempty, compact, and $E_{h}$-invariant. The $2 \times 2$ transition matrix $\mathscr{A}$ is associated with $\Lambda$ via

$$
\mathscr{A}_{i j}= \begin{cases}1 & \text { if } E_{h}\left(I_{i}\right) \supset I_{j} \\ 0 & \text { otherwise }\end{cases}
$$

By (4.3) $\mathscr{A}$ is the matrix given in the statement of the theorem.

For $x \in \Lambda$ we define $\varphi(x) \in \Sigma_{\mathscr{A}}$ by $(\varphi(x))_{i}=j$ if $E_{h}^{i}(x) \in I_{j}, i \geq 0, j=$ 1, 2. The map $\varphi: \Lambda \rightarrow \Sigma_{\mathscr{A}}$ is easily seen to be continuous and commute with the shift $\sigma_{\mathscr{A}}$. That $\varphi$ is surjective follows from the definition of $\mathscr{A}$.

Finally, note that the largest eigenvalue of $\mathscr{A}$ is $\lambda=\frac{1+\sqrt{5}}{2}$, hence the topological entropy of $\left(\Sigma_{\mathscr{A}}, \sigma_{\mathscr{A}}\right)$ is $h\left(\sigma_{\mathscr{A}}\right)=\ln \left(\frac{1+\sqrt{5}}{2}\right)$.

To construct the intervals $I_{1}, I_{2}$, consider the graph of $E_{h}(x)$ in Figure 2. Let $b_{1}$ denote the unique preimage of the fixed point $x=1$ satisfying $b_{1}<0$. Next observe that there is a unique point $a_{1}<0$ such that $E_{h}\left(a_{1}\right)=0$ and that $a_{1}$ has a unique preimage $a_{2}$ with $a_{2}>0$. Finally let $I_{1}=\left[a_{1}, b_{1}\right]$ and $I_{2}=\left[a_{2}, 1\right]$. Then by construction $E_{h}\left(I_{1}\right) \supset I_{2}$ and $E_{h}\left(I_{2}\right) \supset I_{1} \cup I_{2}$.

Remark. An upper bound on the topological entropy of $E_{h}$ is $\ln (2)$. This is most easily seen by complexifying the map $E_{h}$, i.e., $E_{h}(z)=z+h\left(1-\frac{1}{z}\right)$. This is a meromorphic map of the Riemann sphere of degree 2 , and hence has topological entropy $h\left(E_{h}(z)\right)=\ln 2($ see $\S 5)$. The map $E_{h}(x)$ is a subsystem of $E_{h}(z)$, and hence has topological entropy bounded above by $\ln 2$.

One might hope to do somewhat better by using a higher order method. We recall that the second order Runge-Kutta method for a single first order 
autonomous ODE $\dot{x}=f(x)$ is defined by $x_{n+1}=x_{n}+\frac{h}{2}[f(x)+f(x+h f(x))]$. Applying this to equation (4.1) we obtain the map

$$
x \stackrel{R_{h}}{\longrightarrow} x+h\left(1-\frac{1}{2 x}-\frac{x}{2\left(x^{2}+h x-h\right)}\right) .
$$

Theorem 4.2. For all $h>0$ the nonwandering set of the map $R_{h}$ of (4.4) contains a subset $\Lambda$ such that $\left.R_{h}\right|_{\Lambda}$ is topologically semiconjugate to the onesided shift on three symbols, i.e., the subshift of finite type with transition matrix

$$
\mathscr{B}=\left[\begin{array}{lll}
1 & 1 & 1 \\
1 & 1 & 1 \\
1 & 1 & 1
\end{array}\right] \text {. }
$$

$\left(\Sigma_{\mathscr{B}}, \sigma_{\mathscr{B}}\right)$ has topological entropy $h\left(\sigma_{\mathscr{B}}\right)=\ln (3)$, hence $h\left(R_{h}\right) \geq \ln (3)$.

Note that the topological entropy is larger than for $E_{h}$ despite the fact that $R_{h}$ is a higher order method.

Proof. The graph of $R_{h}$ is shown in Figure 3. Note that in addition to the unstable fixed point at $x=1$ the map possesses two additional unstable fixed points at $x=\frac{-h \pm \sqrt{h^{2}+2 h}}{2}$ as well as two additional singular points at $x=\frac{-h \pm \sqrt{h^{2}+4 h}}{2}$. Let $J_{1}=\left[\frac{-h-\sqrt{h^{2}+4 h}}{2}, 0\right], J_{2}=\left[0, \frac{-h+\sqrt{h^{2}+4 h}}{2}\right]$, and $J_{3}=\left[\frac{-h+\sqrt{h^{2}+4 h}}{2}, 1\right]$. Notice that $R_{h}$ maps the interior of each interval $J_{i}$ onto $\mathbf{R}$. Now let $a_{1}=$ $\frac{-h-\sqrt{h^{2}+2 h}}{2}$ be the smallest fixed point of $R_{h}$ and for $i=2,3$ define $a_{i}$ as the unique preimage of $a_{1}$ lying in $J_{i}$ (see Figure 3). Next let $b_{3}=1$ be the largest fixed point of $R_{h}$ and for $k=1,2$ define $b_{k}$ as the unique preimage of $b_{3}$ lying in $J_{k}$. Finally define $I_{k}=\left[a_{k}, b_{k}\right], k=1,2,3$. Then for $k=1,2,3$ we have $R_{h}\left(I_{k}\right) \supset I_{1} \cup I_{2} \cup I_{3}$.

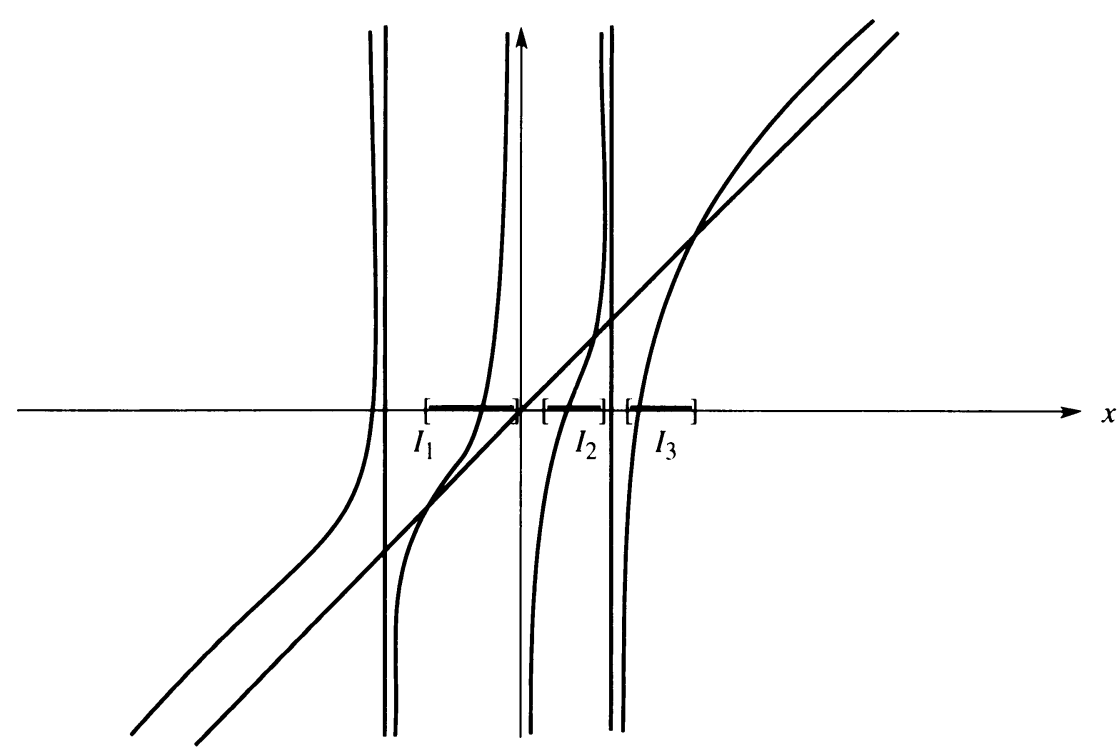

FIGURE 3. Construction of the intervals $I_{1}, I_{2}, I_{3}$. 
We wish to remark that the second order Runge-Kutta algorithm we have examined is one member of the one-parameter family of second order algorithms given by

$$
x_{n+1}=x_{n}+h\left[s f\left(x_{n}\right)+(1-s) f\left(x_{n}+\frac{h}{2(1-s)} f\left(x_{n}\right)\right)\right],
$$

where $0 \leq s<1$ is the parameter (see, e.g., Lambert [L]). The choice $s=\frac{1}{2}$ yields the algorithm we have studied in Theorem 4.2.

Applying this algorithm to the ODE (4.1) we obtain the two-parameter family of maps

$$
x \stackrel{R_{h, s}}{\longrightarrow} x+h\left(1-\frac{s}{x}-\frac{(1-s)^{2} x}{(1-s) x^{2}+h x / 2-h / 2}\right) .
$$

For any $h>0$ and $0 \leq s<1$, this map possesses unstable fixed points at $x=1$ and $x_{1}^{ \pm}(s)=\frac{-h \pm \sqrt{h^{2}+8 h s(1-s)}}{4(1-s)}$ and singular points at $x=0$ and $x_{2}^{ \pm}(s)=\frac{-h \pm \sqrt{h^{2}+8 h(1-s)}}{4(1-s)}$. Observe that for $h>0$ and $0 \leq s<1$ we have $x_{2}^{-}(s)<x_{1}^{-}(s)<0<x_{1}^{+}(s)<x_{2}^{+}(s)<1$, and the graph of $R_{h, s}$ is qualitatively the same as Figure 3. Thus for any $0 \leq s<1$ we can construct intervals $I_{1}, I_{2}$, and $I_{3}$ in essentially the same way as in the proof of Theorem 4.2, and the conclusions of Theorem 4.2 will hold for any member of this one-parameter family of second order Runge-Kutta algorithms.

\section{AN ANALYSIS ON THE RIEMANN SPHERE}

The chaotic behavior observed in the above cases is related in part to the presence of a singularity in the vector field. Some additional insight can be gained by exploring the numerical dynamics of the system

$$
\dot{z}=f(z) \text {, }
$$

where $f$ is a meromorphic function on the Riemann sphere $\bar{C}=C \cup\{\infty\}$ of degree at least two. Thus $f(z)=p(z) / q(z)$ where $p$ and $q$ are polynomials, the degree of $f$ is $\operatorname{deg}(f)=\max \{\operatorname{deg}(p), \operatorname{deg}(q)\}$, and we assume that $\operatorname{deg}(f) \geq 2$. Although our development allows for meromorphic vector fields, it is important to note that our results hold in the special case that $f$ is a polynomial. Thus, in particular, the vector fields we study in this section do not necessarily possess singularities.

We begin by recalling some results from complex analytic dynamics. See Blanchard [Bl] or Devaney [D] for a more complete discussion of this material.

A point $z$ is a periodic point for a mapping $F$ of $\bar{C}$ if there exists an integer $n>0$ such that $F^{n}(z)=z$. Here $F^{n}$ denotes the composition of $F$ with itself $n$ times. The least such $n$ is called the period of $z$. Of course if $n=1$ we call $z$ a fixed point of $F$. A periodic point of period $n$ is attracting (resp. superattracting) if $\left|\left(F^{n}\right)^{\prime}(z)\right|<1$ (resp. $\left|\left(F^{n}\right)^{\prime}(z)\right|=0$ ). If $F(z)$ is a polynomial then $\infty$ is a superattracting fixed point for $F$. A periodic point of period $n$ is repelling if $\left|\left(F^{n}\right)^{\prime}(z)\right|>1$. The Julia set of $F$, denoted $J(F)$, is the closure of the set of repelling periodic points of $F$. The Julia set of a rational map is always nonempty, compact (in $\bar{C}$ ) and completely invariant $\left(z \in J(F)\right.$ implies $F(z) \in J(F)$ and $\left.F^{-1}(z) \subset J(F)\right)$. Moreover, the Julia set is a perfect set (hence it is uncountable). 
The dynamics of $F$ restricted to the Julia set are extremely complicated. Indeed, the topological entropy of $F$ is $h(F)=\ln (\operatorname{deg}(F))$ (see [Bo] and Newhouse [N]). Moreover, if $F$ is a polynomial and the Julia set of $F$ is disconnected, then $\left.F\right|_{J(F)}$ is conjugate to a shift on $n=\operatorname{deg}(F)$ symbols.

Consider now applying any Runge-Kutta numerical method $R_{h}$ to the system (5.1). For meromorphic $f(z)$ with $\operatorname{deg}(f) \geq 2$, the resulting map $z \mapsto R_{h}(z)$ will be a rational map of $\bar{C}$ of degree at least two. The above results from complex dynamics easily yield the following conclusion.

Proposition 5.1. Any Runge-Kutta algorithm $z \mapsto R_{h}(z)$ applied to the system (5.1) has topological entropy $h\left(R_{h}\right) \geq \ln 2$.

Remark. This result is independent not only of the stepsize $h$ but also of the vector field $f$. In particular, the result holds for polynomial vector fields $f$, so the presence of a singularity is not required in order that numerical dynamics exhibit chaotic behavior. More can be said. If one applies Euler's method to $\dot{z}=p(z)$ where $p(z)$ is a polynomial of degree $d$, then the resulting map has degree $d$, hence topological entropy $\ln (d)$. Applying second order RungeKutta yields a map of degree $d^{2}$, thus doubling the topological entropy.

An issue we have not addressed is the effect of roundoff error. Again there is insight to be gained by considering systems on the Riemann sphere. The Julia set has the property that if $z \in J(F)$ and if $U$ is any neighborhood of $z$, then the set $\bar{C}-\bigcup_{i=0}^{\infty} F^{i}(U)$ contains at most two points. Thus the forward iterates $\bigcup_{i=0}^{\infty} F^{i}(U)$ of any neighborhood of a point in $J(F)$ cover virtually the entire Riemann sphere. If one uses a numerical method $N$ to obtain a numerical solution to an initial value problem $\dot{z}=f(z), z(0)=z_{0}$, and if the iterates $\left\{N^{n}\left(z_{0}\right)\right\}$ come close to the Julia set of $N$, then a small roundoff error can cause the numerical solution to diverge more than one would expect looking only at truncation and roundoff error.

It is tempting to think that one can always offset this effect by reducing the stepsize by an appropriate amount. This is not generally the case, however. Indeed, any unstable equilibrium point for $\dot{z}=f(z)$ will be an unstable fixed point for the numerical scheme $N(z)$ and will therefore be an element of the Julia set of $N$. We wish to note that the situation in our setting (numerical approximations to flows) is rather different than in the case of Newton's method applied to polynomials (see, e.g., Smale [Sm], Sutherland [Sut]). For Newton's method one expects generally that the Julia set will have measure zero and the basins of any periodic sinks will have relatively small measure. (The issue of whether the Julia set can have positive measure without being all of $\bar{C}$ is not completely resolved.) Moreover, $z=\infty$ is a repellor for Newton's method (applied to a polynomial), so in general one expects to converge to a root after making a relatively small number of choices of starting value.

Let us consider, on the other hand, the behavior at $z=\infty$ of the planer system

$$
\dot{z}=a_{0}+a_{1} z+\cdots+a_{d} z^{d}=p(z),
$$

where $d=\operatorname{deg} p(z) \geq 2$. If we make the change of coordinates $w=\frac{1}{z}$ then

$$
\dot{w}=-\left(a_{0} w^{2}+a_{1} w+\cdots+a_{d} / w^{d-2}\right),
$$

so we see that $z=\infty$ is not an equilibrium point for $(*)$. The numerical 
behavior of $(*)$, however, has a rather different character. Indeed, for $p(z)$ a polynomial of degree at least 2 and any Runge-Kutta algorithm $R$, the map $z \mapsto R(z)$ is given by a polynomial and thus has $z=\infty$ as a superattracting fixed point. Hence $R(z)$ always has an open set of initial conditions that lie in the basin of $\infty$.

One possible way to address this problem is to use a variable stepsize method, i.e., allow $h=h(z)$ to vary depending on $z$. The above discussion shows, however, that one cannot choose $h(z)$ to be a rational function of $z$ since the resulting numerical scheme is also a rational map.

\section{CONClusion}

We have examined the global dynamical behavior of Euler's method and second order Runge-Kutta when applied to a single ordinary differential equation possessing a singularity at the origin. In both cases the resulting numerical dynamics exhibit chaotic behavior in the form of a subshift of finite type with positive topological entropy. A somewhat surprising result, however, is that the topological entropy of the dynamics for second order Runge-Kutta exceeds that of Euler's method.

An analysis in the complex setting using techniques from complex analytic dynamics reveals that increasing the order of a Runge-Kutta method can increase the degree of the mapping, and hence the topological entropy. Moreover, in the complex setting with a polynomial vector field $p(z)$ of degree at least 2 we have seen that $\infty$ cannot be an equilibrium point for the flow. For any Runge-Kutta scheme, however, $\infty$ is a superattracting fixed point and thus possesses a basin of attraction which is an open set in $\bar{C}$.

In attempting to understand the global dynamics of a given complicated dynamical system one often wishes to integrate a system of ODE's for a long period of time with many choices of initial data. For some systems (e.g., gradient systems) the global behavior of the numerics can be expected to be fairly representative of the dynamics of the underlying system (at least in any compact region of the phase space). The same is true, of course, of the local picture near a stable equilibrium point, but as our examination of systems in the complex plane shows one can expect that the global numerics are not representative of the original system of ODE's. A better understanding of the dichotomy of behaviors between flows arising from polynomial vector fields on $\bar{C}$ on the one hand, and their corresponding numerics on the other, should provide information which helps in devising strategies for dealing with the anomolous behavior we observe in Runge-Kutta algorithms.

\section{ACKNOWLEDGMENT}

The author wishes to thank the referee for pointing out the one-parameter family of second order Runge-Kutta methods discussed at the end of $\S 4$.

\section{REFERENCES}

[BS] S. Batterson and J. Smillie, The dynamics of Rayleigh quotient iteration, SIAM J. Numer. Anal. 26 (1989), 624-636.

[BI] P. Blanchard, Complex analytic dynamics on the Riemann sphere, Bull. Amer. Math. Soc. (N.S.) 11 (1984), 85-141. 
[Bo] R. Bowen, On axiom A diffeomorphisms, CBMS Regional Conf. Ser. in Math., vol. 35, Amer. Math. Soc., Providence, RI, 1978.

[CGS] J. Curry, L. Garnett, and D. Sullivan, On the iteration of a rational function: computer experiments with Newton's method, Comm. Math. Phys. 91 (1983), 267-277.

[D] R. Devaney, An introduction to chaotic dynamical systems, Benjamin/Cummings, Menlo Park, 1986

[Ga] F. R. Gantmacher, The theory of matrices, Vol. II, Chelsea, New York, 1960.

[HS] M. Hirsch and S. Smale, Differential equations, dynamical systems and linear algebra, Academic Press, New York, 1974.

[Ho] K. Hockett, Chaotic numerics from an integrable Hamiltonian system, Proc. Amer. Math. Soc. 108 (1990), 271-281.

[L] J. D. Lambert, Computational methods in ordinary differential equations, Wiley, New York, 1973

[Lo] E. Lorenz, Computational chaos-a prelude to computational instability, Phys. D 35 (1989), 299-317.

[N] S. Newhouse, Entropy and volume, Ergodic Theory Dynamical Systems 8 (1988), 283-299.

[Sh] M. Shub, Global stability of dynamical systems, Springer-Verlag, New York, 1987.

[SV] M. Shub and A. Vasquez, Some linearly induced Morse-Smale systems, the QR algorithm and the Toda lattice, The Legacy of Sonya Kovalevskaya (Linda Keen, ed.), Contemporary Math., vol. 64, Amer. Math. Soc., Providence, RI, 1987.

[Sm] S. Smale, On the efficiency of algorithms of analysis, Bull. Amer. Math. Soc. (N.S.) 13 (1985), $87-121$.

[Sut] S. Sutherland, Finding roots of complex polynomials with Newton's method, Ph.D. Thesis, Boston Univ., Boston, 1989.

[SwYG] P. K. Sweby, H. C. Yee, and D. F. Griffiths, On spurious steady-state solutions of explicit Runge-Kutta schemes, NASA Ames Research Center, preprint, 1990.

Department of Mathematics, The George Washington University, Washington, D.C. 20052 